\title{
Cutoff-independent regularization of four-fermion interactions for color superconductivity
}

\author{
R. L. S. Farias, ${ }^{1}$ G. Dallabona,${ }^{1}$ G. Krein, ${ }^{1}$ and O. A. Battistel ${ }^{2}$ \\ ${ }^{1}$ Instituto de Física Teórica, Universidade Estadual Paulista, Rua Pamplona 145, 01405-900 São Paulo, SP, Brazil \\ ${ }^{2}$ Departamento de Física, Universidade Federal de Santa Maria, 97119-900 Santa Maria, RS, Brazil
}

(Received 31 October 2005; published 27 January 2006)

\begin{abstract}
We implement a cutoff-independent regularization of four-fermion interactions to calculate the colorsuperconducting gap parameter in quark matter. The traditional cutoff regularization has difficulties for chemical potentials $\mu$ of the order of the cutoff $\Lambda$, predicting in particular a vanishing gap at $\mu \sim \Lambda$. The proposed cutoff-independent regularization predicts a finite gap at high densities and indicates a smooth matching with the weak coupling QCD prediction for the gap at asymptotically high densities.
\end{abstract}

DOI: 10.1103/PhysRevC.73.018201

PACS number(s): 24.85.+p, 12.38.Mh, 21.65.+f, 26.60.+c

The study of the properties of high-density quark matter has attracted great interest recently-for reviews and extensive lists of references see Refs. [1]. The earlier studies [2] on this subject found energy gaps of the order of a few MeVs. Since gaps of this order are much too small to have observable consequences, not much attention was given to the subject until recently, when it was shown $[3,4]$ within the context of instanton-motivated four-fermion interactions that gaps of the order of $100 \mathrm{MeV}$ could be obtained. The possibility of gaps of this order were corroborated by subsequent study [5] using weak coupling renormalization group techniques for QCD. The result of Ref. [5] for the two-flavor spin-0 superconducting gap $\Delta$ can be written as

$$
\Delta \sim \frac{\mu}{g^{5}} \exp \left(-\frac{3 \pi^{2}}{\sqrt{2} g}\right)
$$

where $\mu$ is the chemical potential and $g=g(\mu)$ is the QCD coupling constant. This result is clearly nonperturbative but was derived by assuming weak coupling, an assumption likely to be valid only at very high densities. Although inapplicable for densities typically found in the interiors of neutron stars, it seems to be a sound prediction for the color superconducting gap at asymptotically high baryon number densities. In particular, using the one-loop running of $g(\mu)$ with $\mu$, Eq. (1) predicts that $\Delta(\mu)$ is an increasing function of $\mu$. Elaborations and corrections [6] to Eq. (1) do not change this behavior.

In view of the inapplicability of weak coupling techniques at densities of phenomenological interest and the fact that nonperturbative lattice techniques are not yet sufficiently developed to deal with such problems, the use of phenomenological models seem to be necessary to make progress in the field. In this context, models with nonrenormalizable four-fermion interactions have been extensively used to study different aspects of dynamical chiral symmetry breaking $(\mathrm{D} \chi \mathrm{SB})$ and high-density quark matter [7]. However, four-fermion models at the one-loop level predict vanishing superconducting gaps at high densities, a feature that is caused by the use of a regularizing momentum cutoff $\Lambda$ of the divergent one-loop integrals [8-10]. Since the phenomenon of superconductivity involves momenta of the order of the Fermi momentum $k_{F}$, for baryon number densities such that $k_{F} \sim \Lambda$ the cutoff regularization clearly becomes inadequate and the vanishing of the gap at high densities might not be a physical feature of the problem. Although the QCD prediction of Eq. (1) of a nonzero gap is valid only at very high densities, there seems to be no physical motivation for expecting vanishing gaps at moderately high densities. Of course, in principle one cannot exclude the possibility that the spin- 0 gap (considered here) indeed vanishes at intermediate values of the chemical potential and then is again different from zero at much higher densities, as predicted by QCD — but such a question cannot be answered without a detailed nonperturbative calculation within QCD.

The aim of this Brief Report is to present an alternative to the cutoff regularization of pointlike four-fermion interactions. Specifically, the superconducting one-loop integrals are reorganized through mathematical identities in such a way that finite integrals become separated from $\mu$-independent divergent integrals. The finite integrals are integrated without imposing any restriction to the integration momenta and the divergent integrals are related to physical quantities at the $\mathrm{D} \chi \mathrm{SB}$ scale in vacuum. As a result, one obtains a superconducting gap that is nonvanishing at high baryon number densities. We will keep the discussion very general, and no specific four-fermion model will be used to explain our procedure; commitment to a specific model will be made only when presenting numerical results.

Invariably, at the one-loop level pointlike four-fermion interactions of massless quarks lead to a gap equation for the superconducting gap $\Delta$ of the form

$$
1=\lambda G i \int \frac{d^{4} k}{(2 \pi)^{4}}\left[\frac{1}{k_{0}^{2}-(k+\mu)^{2}-\Delta^{2}}+(\mu \rightarrow-\mu)\right],
$$

where $\lambda$ is a numerical coefficient that depends on the Lorentz, color, and flavor structure of the interaction, and $G$ is the strength of the four-fermion interaction. Obviously, this integral is ultraviolet divergent and a regularization scheme must be specified to proceed. The traditional way to regularize the integral of Eq. (2) is via a three- or four-dimensional sharp cutoff, or via a form factor. In view of the nonrenormalizable nature of the interaction, the regularization becomes part of the model. Using a three-dimensional sharp cutoff, the self-consistent solution for $\Delta=\Delta(\mu)$ leads to the result that 
$\Delta=0$ for $\mu \sim \Lambda$; the use of a form factor instead leads to the same qualitative behavior.

The regularization method we advocate here, proposed in Ref. [11] and used in different contexts [12], avoids the use of an explicit regulator and the calculation of any divergent integrals. Specifically, instead of introducing a cutoff in the integrals of Eq. (2), the integrands are manipulated in such a way that divergences are isolated in well-known, $\mu$-independent one-loop divergent integrals that can be related to divergent integrals of the problem of $\mathrm{D} \chi \mathrm{SB}$ in vacuum. Finite integrals are integrated without imposing any restriction on their integrands and the remaining divergent integrals are fitted to physical quantities at the $\mathrm{D} \chi \mathrm{SB}$ scale in vacuum.

This is done in the following way. To safely manipulate the divergent integrals, we initially assume the integral in Eq. (2) regularized by some regulating function $f\left(k^{2} / \Lambda^{2}\right)$, where $\Lambda$ is the regularization parameter. We rewrite the gap equation as

$$
1=\lambda G i \int_{\Lambda} \frac{d^{4} k}{(2 \pi)^{4}}\left[\frac{1}{k_{0}^{2}-(k+\mu)^{2}-\Delta^{2}}+(\mu \rightarrow-\mu)\right],
$$

where $\int_{\Lambda}$ indicates that the integral is regularized. The next step is the reorganization of the integrands as previously mentioned. This goal can be achieved by using the identity

$$
\begin{aligned}
\frac{1}{k_{0}^{2}-(k \pm \mu)^{2}-\Delta^{2}} & \\
= & \frac{1}{k_{0}^{2}-k^{2}-\Delta^{2}}-\frac{\left(\mp 2 k \mu-\mu^{2}\right)}{\left(k_{0}^{2}-k^{2}-\Delta^{2}\right)^{2}}+\frac{\left(\mp 2 k \mu-\mu^{2}\right)^{2}}{\left(k_{0}^{2}-k^{2}-\Delta^{2}\right)^{3}} \\
& -\frac{\left(\mp 2 k \mu-\mu^{2}\right)^{3}}{\left(k_{0}^{2}-k^{2}-\Delta^{2}\right)^{3}\left[k_{0}^{2}-(k \pm \mu)^{2}-\Delta^{2}\right]}
\end{aligned}
$$

which can be obtained after using three times in succession the identity

$$
\begin{aligned}
& \frac{1}{k_{0}^{2}-(k \pm \mu)^{2}-\Delta^{2}}=\frac{1}{k_{0}^{2}-k^{2}-\Delta^{2}} \\
& +\frac{\mu^{2} \pm 2 k \mu}{\left(k_{0}^{2}-k^{2}-\Delta^{2}\right)\left[k_{0}^{2}-(k \pm \mu)^{2}-\Delta^{2}\right]}
\end{aligned}
$$

This identity corresponds to a subtraction of the original expression at the point $\mu=0$, akin to what is done with nonconvergent integrals in dispersion relations. When the identity of Eq. (4) is substituted into Eq. (3), one can rewrite the gap equation in the form

$$
\begin{aligned}
1= & 8 \lambda G\left\{2\left[i I_{\text {quad }}\left(\Delta^{2}\right)\right]-4 \mu^{2}\left[i I_{\log }\left(\Delta^{2}\right)\right]\right. \\
& \left.+I_{\text {fin }}\left(\Delta^{2}, \mu\right)+I_{\text {fin }}\left(\Delta^{2},-\mu\right)\right\},
\end{aligned}
$$

where

$$
\begin{aligned}
I_{\text {quad }}\left(\Delta^{2}\right) & =\int_{\Lambda} \frac{d^{4} k}{(2 \pi)^{4}} \frac{1}{k_{0}^{2}-k^{2}-\Delta^{2}}, \\
I_{\log }\left(\Delta^{2}\right) & =\int_{\Lambda} \frac{d^{4} k}{(2 \pi)^{4}} \frac{1}{\left(k_{0}^{2}-k^{2}-\Delta^{2}\right)^{2}},
\end{aligned}
$$

$$
\begin{aligned}
I_{\text {fin }}\left(\Delta^{2}, \mu\right)= & i \int \frac{d^{4} k}{(2 \pi)^{4}} \frac{1}{\left(k_{0}^{2}-k^{2}-\Delta^{2}\right)^{3}} \\
& \times\left[2 \mu^{2}\left(\mu^{2}-4 \Delta^{2}\right)+\frac{\left(\mu^{2}+2 k \mu\right)^{3}}{\left(k_{0}^{2}-(k+\mu)^{2}-\Delta^{2}\right)}\right] .
\end{aligned}
$$

In the absence of the regularizing function, $I_{\text {quad }}\left(\Delta^{2}\right)$ and $I_{\log }\left(\Delta^{2}\right)$ are divergent, but $I_{\text {fin }}\left(\Delta^{2}, \mu\right)$ is finite. The divergent integrals are of the same form as those appearing in the problem of $\mathrm{D} \chi \mathrm{SB}$ in vacuum, with the difference that the mass scale appearing in the superconductivity integrals is $\Delta$ whereas in the $\mathrm{D} \chi \mathrm{SB}$ integrals the scale is the constituent quark mass $M$. But one can relate the integrals at different mass scales by making use of the following scaling properties [11,12]:

$$
\begin{aligned}
I_{\text {quad }}\left(\Delta^{2}\right)= & I_{\text {quad }}\left(M^{2}\right)+\left(\Delta^{2}-M^{2}\right) I_{\log }\left(M^{2}\right) \\
& +\frac{i}{(4 \pi)^{2}}\left[\Delta^{2}-M^{2}-\Delta^{2} \ln \left(\frac{\Delta^{2}}{M^{2}}\right)\right] \\
I_{\log }\left(\Delta^{2}\right)= & I_{\log }\left(M^{2}\right)-\frac{i}{(4 \pi)^{2}} \ln \left(\frac{\Delta^{2}}{M^{2}}\right) .
\end{aligned}
$$

These can be obtained by integrating the relations

$$
\frac{\partial I_{\text {quad }}\left(\mu^{2}\right)}{\partial \mu^{2}}=I_{\log }\left(\mu^{2}\right), \quad \frac{\partial I_{\log }\left(\mu^{2}\right)}{\partial \mu^{2}}=\frac{-i}{16 \pi^{2} \mu^{2}},
$$

between the two mass scales $M^{2}$ and $\Delta^{2}$. The expressions in Eq. (12) can be proven by direct differentiation of the integrals in Eqs. (7) and (8). In integrating these equations, finite integrals are integrated without regularization. The relations of Eqs. (10) and (11) allow us to normalize the divergent integrals $I_{\text {quad }}\left(\Delta^{2}\right)$ and $I_{\log }\left(\Delta^{2}\right)$ to chiral observables at the scale of the constituent quark mass $M$. This is so because $I_{\text {quad }}\left(M^{2}\right)$ and $I_{\log }\left(M^{2}\right)$ in this class of models are related, respectively, to the chiral condensate $\langle\bar{\psi} \psi\rangle$ and to the pion decay constant $f_{\pi}$ (in the chiral limit) by

$$
i I_{\text {quad }}\left(M^{2}\right)=\frac{-\langle\bar{\psi} \psi\rangle}{12 M}, \quad i I_{\log }\left(M^{2}\right)=-\frac{f_{\pi}^{2}}{12 M^{2}} .
$$

Therefore, making use of the relations of Eqs. (10) and (11), one can eliminate $I_{\text {quad }}\left(\Delta^{2}\right)$ and $I_{\log }\left(\Delta^{2}\right)$ in the gap equation for $\Delta$ in favor of $\langle\bar{\psi} \psi\rangle$ and $f_{\pi}$, obtaining

$$
\begin{aligned}
1= & \lambda G\left[2 I_{1}\left(M^{2}, \Delta^{2}\right)-4 \mu^{2} I_{2}\left(M^{2}, \Delta^{2}\right)\right. \\
& \left.+I_{\text {fin }}\left(\Delta^{2}, \mu\right)+I_{\text {fin }}\left(\Delta^{2},-\mu\right)\right],
\end{aligned}
$$

where

$$
\begin{aligned}
I_{1}\left(M^{2}, \Delta^{2}\right)= & -\frac{\langle\bar{\psi} \psi\rangle}{12 M}-\left(\Delta^{2}-M^{2}\right) \frac{f_{\pi}^{2}}{12 M^{2}} \\
& -\frac{1}{(4 \pi)^{2}}\left[\Delta^{2}-M^{2}-\Delta^{2} \ln \left(\frac{\Delta^{2}}{M^{2}}\right)\right], \\
I_{2}\left(M^{2}, \Delta^{2}\right)= & \frac{f_{\pi}^{2}}{12 M^{2}}-\frac{1}{(4 \pi)^{2}} \ln \left(\frac{\Delta^{2}}{M^{2}}\right) .
\end{aligned}
$$

In this way, we have eliminated the divergences without specifying any regularization function. The use of $f\left(k^{2} / \Lambda^{2}\right)$ is simply a matter of formality, in the sense that once the integrals are regularized, they can be freely manipulated. 
In a renormalizable theory, one would simply do the same manipulations but the remaining divergences would be eliminated by counterterms added to the original Lagrangian (or Hamiltonian). The basic divergent integrals could also be written in terms of an arbitrary mass scale $\hat{m}$, which can be used to relate observables at different mass scales by using the standard renormalization group techniques. The entire regularization-renormalization process can be done without specifying any regularization function and without calculating any divergent integral. In the present case with nonrenormalizable interactions, the divergences are simply fitted to physical quantities at the chiral symmetry mass scale $M$. Note that instead of referring to a regularizing function $f\left(k^{2} / \Lambda^{2}\right)$ parametrized in terms of a momentum scale $\Lambda$, one could have simply used dimensional regularization, fit the divergent integrals to $\langle\bar{\psi} \psi\rangle$ and $f_{\pi}$ as done here, and evaluate finite integrals at the physical dimension.

So far, the discussion has been completely general, valid for any pointlike four-fermion interaction. To see the physical consequences of our proposed method, we solve the gap equation for a specific four-fermion model. For illustrative purposes, we use the simple chirally symmetric SU(2) Nambu-Jona-Lasinio model with scalar-plus-pseudoscalar four-fermion interactions [10]. For such a model, $\lambda=8 N_{f}$, where $N_{f}=2$ is the number of flavors. The model needs only two parameters as input: One is the strength of the interaction and the other is a regularization parameter that in the present approach can be taken to be one of the divergent integrals. Using a standard value for the strength of the interaction, $G=3.1 \mathrm{GeV}^{-2}$, and $\langle\bar{\psi} \psi\rangle=(-250 \mathrm{MeV})^{3}$ it is possible to obtain approximately $M=313 \mathrm{MeV}$ and $f_{\pi}=93 \mathrm{MeV}$. With these values, one obtains the solid line in Fig. 1. Clearly, this result shows that the superconducting gap $\Delta$ is an increasing function of the chemical potential $\mu$. Although we have not plotted the result for larger values of $\mu$, the curve actually keeps increasing very slowly as a logarithmic function, and for $\mu \sim 3 \mathrm{GeV}$ the gap is $\Delta \simeq 300 \mathrm{MeV}$. The basic reason for the nonvanishing of $\Delta$ at high values of $\mu$ is that no momentum cutoff is enforced on the finite integral and therefore there is enough phase space at large chemical potentials for allowing larger gaps.

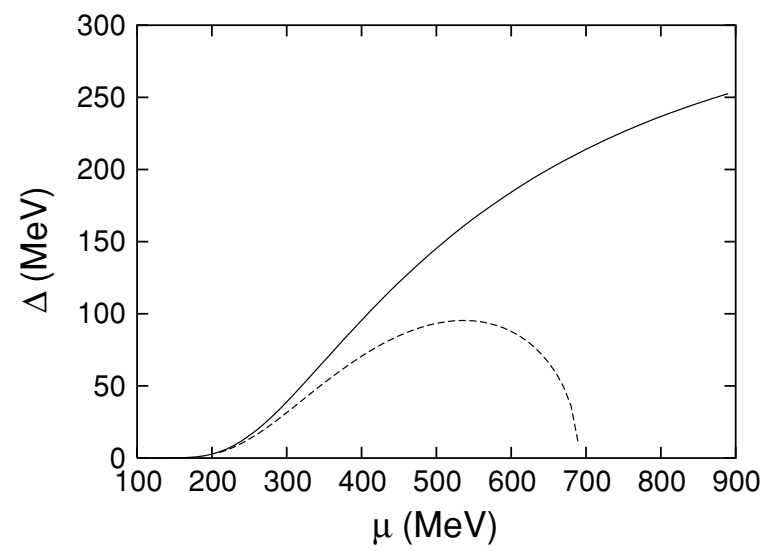

FIG. 1. Superconducting gap $\Delta$ as a function of the quark chemical potential. The solid line is the solution of Eq. (14) and the dashed line is the result using a cutoff in $I_{\text {fin }}$.
To substantiate this we have imposed a cutoff $\Lambda=650 \mathrm{MeV}$ on $I_{\text {fin }}$ and obtained the dashed curve in Fig. 1 . The result is clear: The gap vanishes for chemical potentials of the order of the cutoff $\mu \sim \Lambda$. Note that for consistency one should have used the same cutoff in the finite integrals that appear in the derivation of Eqs. (10) and (11), but this would simply worsen the situation, in the sense that $\Delta$ would be zero for $\mu<700 \mathrm{MeV}$. We repeated the calculation using other types of pointlike four-fermion interactions and obtained qualitatively similar results.

The same scheme can be used at finite temperatures [13]; the gap equation would be given by an expression similar to Eq. (14), with the difference that $I_{\text {fin }}\left(\Delta^{2}, \mu\right)$ would include a term with the Fermi-Dirac distributions for quarks and antiquarks. In connection to this, it is worth mentioning that the integration of finite integrals without imposing a cutoff has been advocated in Refs. [14] in the context of $\mathrm{D} \chi \mathrm{SB}$, where the finite-temperature integrals with the Fermi-Dirac distributions are freely integrated, whereas the divergent integrals are still evaluated with a cutoff $\Lambda$. The crucial difference with the present approach is the use of the scaling relations of Eqs. (10) and (11), by which one can always relate the divergent integrals to vacuum quantities without commitment to a specific regularization. Detailed finite-temperature results using the scheme proposed here will be presented elsewhere [13].

Before concluding, one should notice that although we have not imposed a cutoff on finite integrals, there is still, of course, an implicit regularization scale dependence in the model through the fitting of $I_{\text {quad }}$ and $I_{\log }$ to vacuum physical quantities $\langle\bar{\psi} \psi\rangle$ and $f_{\pi}$ as in Eq. (13). This dependence reflects the fact that the physical quantities (and the coupling $G$ ) are fitted at the implicit regularization scale $\Lambda$. Equivalently, had we used explicit dimensional regularization, a mass scale would enter the problem to match the physical dimensions of the integrals and this scale would also be implicit in the fitting of the divergent integrals. This scale sets a limit on the chemical potential $\mu$ for which the fitting makes physical sense. Therefore, to use a four-fermion model at very high densities one must devise a scheme to extend the model beyond the vacuum scale. This actually can be done using the method proposed in Ref. [8] by making the coupling run with $\Lambda$, that is, by making $G=G(\Lambda)$, and postulating a $\mu$ dependence of $\Lambda$. In Ref. [8] this was done by demanding $\Lambda$ independence of $f_{\pi}$ in a cutoff regularization scheme. In the present approach such a method can be applied using the scaling properties of the divergent integrals $I_{\text {quad }}$ and $I_{\log }$. A detailed discussion on this will be presented in a separate publication [13]. In this context, it would also be interesting to implement the method discussed here in instanton-motivated interactions, where the cutoff has a physical origin and has a density dependence such that the associated form factor peaks around the Fermi momentum with a width of the order of the inverse size of the instanton [15]. Since the instanton effects at large density are suppressed because the effective coupling becomes small, the present method might be useful for handling a possible smooth matching to perturbative QCD.

In summary, we have shown that using a cutoffindependent regularization of nonrenormalizable pointlike four-fermion interactions at the one-loop level one obtains 
nonvanishing superconducting gaps at high densities in quark matter. The result also indicates a smooth matching without abrupt discontinuities with the weak coupling QCD prediction for the gap [5,6]. Although this QCD prediction of a nonzero gap is valid only at asymptotically high quark densities, it seems nevertheless reasonable to assume that the vanishing of the gap at high densities in four-fermion models is a nonphysical artifact of the cutoff regularization. Admittedly, there is an implicit assumption that four-fermion models can be used at energies higher than the chiral symmetry breaking scale. This can be naturally implemented by making regularization parameters density dependent, as physically motivated in Ref. [8]. In this sense, the present approach opens new opportunities for applications of four-fermion models for studying properties of high-density quark matter without facing the difficulties imposed by a cutoff. Obviously, the technique presented here is applicable in a wider context where nonrenormalizable interactions are used for modeling physical systems, such as atomic condensates, for example.

The authors thank Thomas Schäfer and Qun Wang for useful correspondence. This work was partially supported by CNPq and FAPESP (Brazilian agencies).
[1] K. Rajagopal and F. Wilczek, in Handbook of $Q C D$, edited by M. Shifman (World Scientific, Singapore, 2001); M. G. Alford, Annu. Rev. Nucl. Part. Sci. 51, 131 (2001); I. A. Shovkovy, Found. Phys. 35, 1309 (2005); G. Nardulli, Riv. Nuovo Cimento 25N3, 1 (2002); D. H. Rischke, Prog. Part. Nucl. Phys. 52, 197 (2004); M. Buballa, Phys. Rep. 407, 205 (2005); M. Huang, Int. J. Mod. Phys. E 14, 675 (2005); T. Schäfer, hep-ph/0509068.

[2] D. Bailin and A. Love, Phys. Rep. 107, 325 (1984).

[3] M. Alford, K. Rajagopal, and F. Wilczek, Phys. Lett. B422, 247 (1998).

[4] R. Rapp, T. Schäfer, E. V. Shuryak, and M. Velkovsky, Phys. Rev. Lett. 81, 53 (1998).

[5] D. T. Son, Phys. Rev. D 59, 094019 (1999).

[6] T. Schäfer and F. Wilczek, Phys. Rev. D 60, 114033 (1999); R. D. Pisarski and D. H. Rischke, ibid. 61, 051501(R) (2000); 61, 074017 (2000); D. K. Hong, V. A. Miransky, I. A. Shovkovy, and L. C. R. Wijewardhana, ibid. 61, 056001 (2000); S. D. H. Hsu and M. Schwetz, Nucl. Phys. B572, 211 (2000); W. E. Brown, J. T. Liu, H. C. Ren, Phys. Rev. D 61, 114012 (2000); D. H. Rischke, ibid. 64, 094003 (2001); Q. Wang and D. H. Rischke, ibid. 65, 054005 (2002).

[7] T. Hatsuda and T. Kunihiro, Phys. Rev. Lett. 55, 158 (1985); V. Bernard, Ulf-G. Meissner, and I. Zahed, Phys. Rev. D 36, 819 (1987); U. Vogl and W. Weise, Prog. Part. Nucl. Phys. 27, 195
(1991); T. Hatsuda and T. Kunihiro, Phys. Rep. 247, 221 (1994); M. Buballa, ibid. 407, 205 (2005).

[8] R. Casalbuoni, R. Gatto, G. Nardulli, and M. Ruggieri, Phys. Rev. D 68, 034024 (2003).

[9] F. Gastineau, R. Nebauer, and J. Aichelin, Phys. Rev. C 65, 045204 (2002).

[10] T. M. Schwarz, S. P. Klevansky, and G. Papp, Phys. Rev. C 60, 055205 (1999).

[11] O. A. Battistel, Ph.D. thesis, Universidade Federal de Minas Gerais, Brazil, 1999.

[12] O. A. Battistel and C. Nemes, Phys. Rev. D 59, 055010 (1999); O. A. Battistel and G. Dallabona, Nucl. Phys. B610, 316 (2001); Phys. Rev. D 65, 125017 (2002); J. Phys. G 28, 2539 (2002); Phys. Rev. D 72, 045009 (2005); O. A. Battistel and G. Krein, Mod. Phys. Lett. A 18, 2255 (2003); D. E. Carneiro, A. P. Baeta Scarpelli, M. Sampaio, and M. C. Nemes, J. High Energy Phys. 12 (2003) 044.

[13] R. L. S. Farias, G. Dallabona, G. Krein, and O. A. Battistel, in preparation.

[14] D. Blaschke, Yu. L. Kalinovski, P. Petrow, S. M. Schmidt, M. Jaminon, and B. Van den Bossche, Nucl. Phys. A592, 561 (1995); P. Costa, M. C. Ruivo, and Yu. L. Kalinovsky, Phys. Lett. B577, 129 (2003).

[15] T. Schäfer, Phys. Rev. D 65, 094033 (2002). 